\title{
Industry Requirements and Associated Research Issues in the Extended Enterprise
}

\author{
Jim Browne, \\ CIMRU, \\ University College \\ Galway, \\ Galway, Ireland. \\ Tel: +353.91.750414 \\ Fax: +353.91.562894 \\ Jimmie.Browne@ucg.ie.
}

\author{
Peter Sackett, \\ The CIM Institute, \\ Cranfield University, \\ Bedford, \\ U.K. \\ Tel: +44.1234 .754073$ \\ Fax: +44.1234.750852 \\ Sackett@cim.cran.ac.uk
}

\author{
Hans Wortmann, \\ Technical University of \\ Eindhoven, \\ Eindhoven, \\ The Netherlands. \\ Tel: +31.40 .472290$ \\ Fax: +31.40 .451275$ \\ hwo@bdk.tue.nl
}

\begin{abstract}
In this paper, we consider the difficulties faced by manufacturing companies and their response in terms of the emergence of the Extended Enterprise. We argue that the Extended Enterprise represents the context within which manufacturing systems research must be conducted and we identify what we consider to be the key topics for future manufacturing systems research and development.
\end{abstract}

\section{THE MANUFACTURING SYSTEMS ENVIRONMENT}

Today's manufacturing enterprise operates in a tremendously competitive environment. This competitive environment arises from a series of underlying realities viz:

- Global markets

- Customers demanding high quality, low cost and fast delivery of increasingly customised products - mass customisation.

- The need to develop environmentally benign products and processes. 


\subsection{Global Markets}

Changes in the international trading relationships, manufacturing and information technology and infrastructure developments are leading to the realisation of the global market. In the past, companies operated in local markets, were subject to local cost and pricing structures and competed with local competitors. Trading conditions, customs controls and tariffs provided further barriers to global trade. Today, a vastly superior transport infrastructure, global communications systems, and increasingly open markets (e.g. the recent GATT agreement) have changed all of that.

\subsection{The Rise of Mass Customisation}

With the development of hard automation in the early 20th century (the transfer line etc.) the age of mass produced standard products began. The relatively low cost of such products allowed significant proportions of the population of the western world to acquire consumer goods. The development of information technology, and it's application to manufacturing technology in the 1960s and 1970s changed everything. Automation based on information technology is inherently different to the older "hard" automation in that it is flexible. Computer based automation facilitates the production of a wider range of products in lower volumes economically. Today we are approaching the stage where "mass customisation" is becoming a reality. In short, manufacturers are increasingly required to produce customised products at mass production prices.

\subsection{Environmentally Benign Products and Processes}

Society is putting pressure on manufacturers, to create production systems which are neutral with respect to the environment. This pressure may take the form of legal regulations, economic and marketing requirements.

Today progressive manufacturing companies are developing a total life cycle approach to their products. One multinational supplier of telecommunications equipment and services to the European market has developed a "Product Life Cycle Management" program which currently includes six major activities, namely: design and technology, purchasing of supplies and materials, manufacturing processes, waste reduction and energy management, packaging and post consumer materials management. This company now includes environmental considerations as part of it's supplier qualification process. The "post consumer materials management" activity represents a long term challenge but is based on the following ideas : in the near future, manufacturing will refurbish and reuse products and recycle as much of their contents as possible; finally companies will source new markets for the recycled material and safely dispose of residual materials when necessary.

Tipnis (1993) makes reference to the "expanded responsibility" of the manufacturer over the entire life cycle and talks about the design of products for sustainability. In his view design for sustainability provides specific targets for design for manufacture, assembly, service, disassembly, and recycling. 
In Europe, the Council of the European Union has issued several directives regarding the environmentally sound production, distribution, use and disposal of products. These directives include the principles of the civil liability of the manufacturer for environmental damage caused by his products, financial incentives to achieve effective protection of the environment such as taxes on the use of damaging materials, material disposal charges, independent environmental audits and charges on non-biodegradable materials such as certain plastics. It is likely that in the near future manufacturers will be required to recycle obsolete products themselves or face high public disposal charges.

\section{THE EMERGENCE OF THE EXTENDED ENTERPRISE}

How can a manufacturing company respond to the pressures placed on it by global competition? In the past the response was to make the factory more efficient and responsive by developing CIM (Computer Integrated Manufacturing) solutions. Now, however, the challenge is greater and requires that a degree of integration takes place across the whole value chain.

De Meyer suggests that manufacturing must "see itself as a link in an integrated value added chain, whose goal is to serve the customer" (my emphasis). Coming from a very different perspective, namely that of environmentally benign production, Tipnis (1993) also suggests a similar view when he subtitles his paper "How to design products that are environmentally safe to manufacture/assemble, distribute, use, service/repair, discard/collect, disassemble, recover/recycle and dispose?". It is clear that the manufacturing function must look beyond "the four walls of the manufacturing plant". Global competition and the emerging pressures to develop environmentally benign products and processes, force manufacturing professionals to take a broader view. We term this broader view the "Extended Enterprise".

We believe that the market place to which manufacturing businesses must respond includes:-

- Business processes which cross enterprise boundaries to interface with functional areas in other companies, for example, product design or manufacturing process definition.

- Supplier/customer integration (people and processes) through interchange of commercial/technical data.

- Ability to function effectively as links for information and product in unbuffered supply/distribution chains.

The ability to network the activities of a number of entities to produce and sell manufactured products profitably depends on the relationship of these entities and the communication that passes between them. We are accustomed to thinking about this in the context of a single enterprise with different departments, Sales, Design, Engineering, Manufacturing, Distribution etc. However, within a global market-place, entities from many different enterprises, or entities which in themselves are nominally independent enterprises, relate via a single product to produce a designed result. An example might be a merchandising entity recognising a busi- 
ness opportunity and requesting :-

- a design entity to design it;

- a manufacturing entity to build it;

- a distribution entity to distribute it and;

- a marketing entity to sell it.

The implication of such an example is that all the entities can be considered as "flexible" or "programmable" within their expertise envelope.

This view of the Extended Enterprise is represented in Figures 1 and 2. These figures also serve to illustrate the context for manufacturing research and development. In the past the emphasis was on integration inside the four walls of the manufacturing plant [See Figure 1].

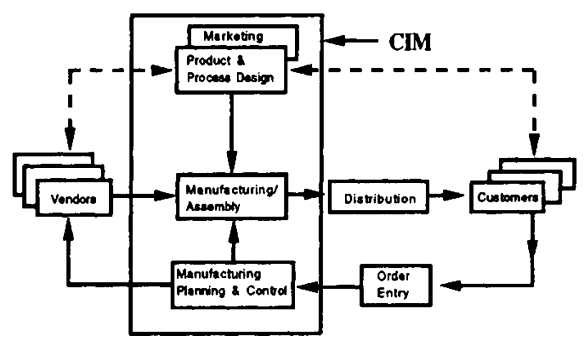

Figure 1 : CIM

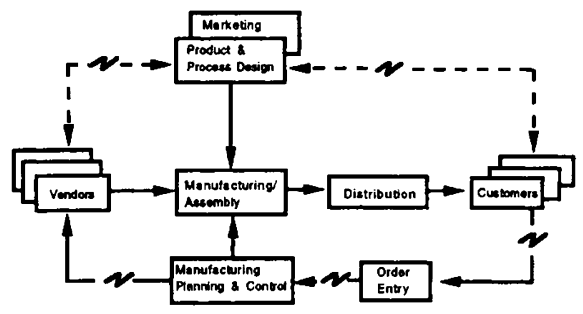

Figure 2 : The Extended Enterprise

CAD/CAM Integration, Integration of Production Planning \& Control Systems, the development of sophisticated manufacturing processes and their control through sophisticated shop floor control systems, formed the agenda. Today the emphasis has changed to include the supply chain (integration of the supply chain through EDI \& JIT), customer driven manufacturing 
including for example, the integration of manufacturing and distribution planning and control systems. In fact this issue of the integration of manufacturing and distribution is a good example. In the past, the two systems were seen to be quite separate and decoupled by warehouses and storage points of various descriptions. (See Figure 3).

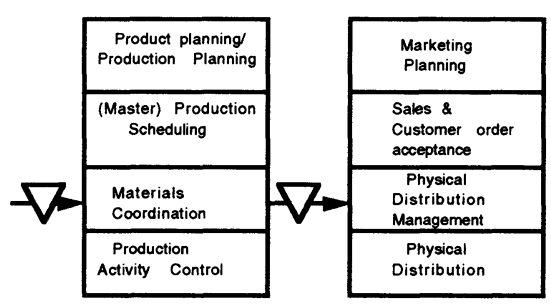

Figure 3 Manufacturing Decoupled from Marketing and Physical

Today with the need to support customization and to realize shorter product delivery lead times, we need to develop integrated systems (See Figure 4).

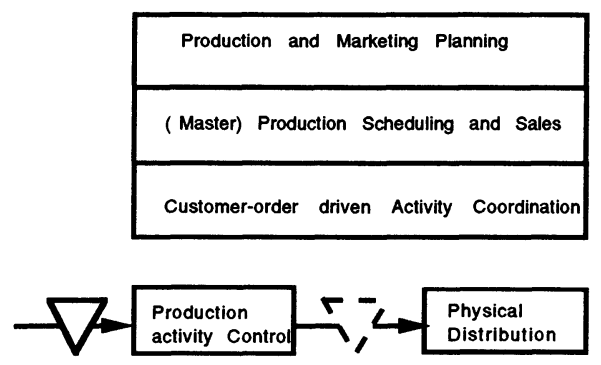

Figure 4 Manufacturing and Physical Distribution driven by Customer Orders.

The emergence of the Extended or Networked Enterprise is of course facilitated by today's emerging computing and telecommunications technologies. EDI is one such technology as indicated in Figure 2. (See Browne, Sackett and Wortmann, 1994). 


\section{THE EXTENDED ENTERPRISE-EVOLUTION OR REVOLUTION?}

We believe that this notion of the Extended (or Networked) Enterprise represents in some ways, a logical development of much of the efforts of manufacturing specialists over the past ten years or so. For the past ten years manufacturing systems specialists have evolved a series of approaches to the design and operation of manufacturing systems. In our view, the drive to inter enterprise networking represents an extension and a synthesis of many of these approaches. Approaches, such as MRP, JT, EDI, WCM, Concurrent Engineering, Lean Production, Benchmarking and Business Process Redesign, essentially synthesise into the inter enterprise networking model. In this section we briefly outline these approaches and argue that they culminate in Inter Enterprise networking.

The ideas of MRP, originally developed by Orlicky of IBM in the 1960s were important not least because they taught us the importance of hierarchical planning and the involvement of many department and functions within manufacturing to solve materials planning and control problems. Later in the late 1970 s and early 1980 s, JIT ideas originally developed in the Japanese automotive industry, became known in Europe and the U.S. JIT emphasised customer involvement in the final scheduling of production systems and close co-operation with suppliers to achieve quality and timely delivery. Thus already in the early 1980 s JIT began to focus the view of manufacturing systems specialists on issues outside the four walls of the manufacturing plant, viz. customer requirements of timely delivery and supplier involvement.

In many ways the ideas of the WCM (World Class Manufacturing) school (See for example Schonberger 1990) developed from the experience of JIT implementation studies in the U.S.A. Issues of continuous improvement, training of people, integration of product design and process design to facilitate efficient manufacturing were also emphasised. Hayes, Wheelwright \& Clark for example identified the key characteristics of world class manufacturing as follows :

1. Becoming the best competitor. 'Being better than almost every other company in your industry in at least one aspect of manufacturing'.

2. Growing more rapidly and being more profitable than competitors. World-class companies can measure their superior performance by 'observing how their products do in the marketplace and by observing their cashbox'.

3. Hiring and retaining the best people. 'Having workers and managers who are so skilled and effective that other companies are continually seeking to attract them away from your organisation'.

4. Developing engineering staff. 'Being so expert in the design and manufacture of production equipment that equipment suppliers are continually seeking one's advice about possible modifications to their equipment, one's suggestions for new equipment, and one's agreement to be a test site for one of their pilot models'.

5. Being able to respond quickly and decisively to changing market conditions. 'Being more nimble than one's competitors in responding to market shifts or pricing changes, and in getting new products out into the market faster than they can'.

6. Adopting a product and process engineering approach which maximises the performance 
of both. 'Intertwining the design of a new product so closely with the design of its manufacturing process that when competitors "reverse engineer" the product they find that they cannot produce a comparable one in their own factories without major retooling and redesign expenses'.

7. Continually improving. 'Continually improving facilities, support systems and skills that were considered to be "optimal" or "state of the art" when first introduced, so that they increasingly surpass their initial capabilities'. Hayes, Wheelwright, and Clark also go on to say that 'the emphasis on continual improvement is the ultimate test of a world class organisation'.

Meanwhile the proponents of Concurrent Engineering (CE) begin to emphasise the issue of product design time and to research business, technological and organisational themes in order to reduce time to market It is interesting that one of the important themes in CE is the development of standards to support the exchange of products data between the CAD systems of suppliers and their customers. The work on CE is of course ongoing within the ESPRIT and BRITE-EURAM programmes of the EU and the CALS initiative in the U.S.A.

EDI technology emerged in the late 1980s. Initially used to support business transactions (invoicing, purchase order call off) between suppliers an their customers, it is now beginning to be used to exchange technological product data. Today's most advanced manufacturing companies use EDI to exchange production and purchasing information and to support joint (with suppliers and/or customers) engineering development teams. The STEP initiative is now rapidly moving into industrial practice. Tools are under development to facilitate the use of STEP data including engineering data exchange, data storage and archiving across distributed databases.

Womack et al (1990) defined 'Lean Production' as the successor to mass production. Like mass production, the ideas of lean production were initially developed in the auto industry. Mass production arose in the U.S.A. in the early 20th century in the Ford Motor Company. Lean production developed initially in the Toyota plants in Japan at the end of the 20th century. Clearly our ideas on Lean Production are still under development. According to Jones (1992) the essential characteristics of Lean Production can be summarised as follows :

1. It is customer driven, not driven by the needs of manufacturing.

2. All activities are organised and focused on a product line basis led by a product champion, with functional departments playing a secondary, servicing role.

3. All activities are team based and the organi sation is horizontally and not vertically oriented.

4. The whole system involves fewer actors, all of whom are integrated with each other 330 engineers in the product-development team versus 1400,340 suppliers versus 1500 , about 300 dealer principals versus 3600 (to sell 2 million vehicles) and 2000 assembly employees versus between 3000 and 5500 (for plant assembling 250000 units a year).

5. There is a high level of information exchanged between all the actors and a transparent and real cost structure.

6. The activities are co-ordinated and evaluated by the flow of work through the team or 
the plant, rather than by each department meeting its plan targets in isolation.

7. The discipline necessary for the system to function and expose problems is provided by JT and Total Quality in the plant and supplier and dealer performance evaluation.

8. Wherever possible responsibility is devolved to the lowest level possible, in the plant or to suppliers.

9. The system is based on stable production volumes but with a great deal of flexibility.

10. Relations with employees, suppliers and dealers are based on reciprocal obligations that are the result of treating them as fixed costs.

Thus Lean Production addresses Product Strategy, Product Development, the Supply Chain, Manufacturing and Product Distribution. Essentially it addresses many issues in the value chain which results in a networking of customers, assemblers and suppliers.

In more recent years Benchmarking and Business Process Redesign have emerged. The central idea of benchmarking is to decompose a business into it's essential processes, identify examples of best practice for each of these practices and then define an approach to achieve best practice. Thus benchmarking seeks to improve existing processes. Business process redesign (sometimes termed business process re-engineering) offers a more radical approach. It seeks to identify each business process, question it's relevance to the achievement of business objectives and redesign the overall business to incorporate only appropriate processes. In fact benchmarking may well be used following business process redesign to identify and achieve best practice for each individual process. For a detailed discussion on business process redesign and benchmarking see Hammer and Champy (1993). It is worth noting also that most of the work on benchmarking and business process redesign was done in the U.S.A. Also the software tools for business process redesign come primarily from North America.

Clearly many of the ideas and approaches outlined here support the manufacturing enterprise in it's evolution towards the integrated enterprise model we have defined earlier. However the Integrated Enterprise represents a quantum leap for manufacturing systems. The changes which it requires are so far reaching that, in our view, research and development work is necessary if industry is to be successful in the 21 st century. Our view of the topics which require research and development effort is presented in Section 4 following.

\section{RESEARCH ISSUES IN THE EXTENDED ENTERPRISE}

Here we will try to present the topics which we believe need to be addressed by manufacturing systems researchers, in order to facilitate the emergence of the Extended Enterprise. We will identify the topics through five headings, namely :

- Inter-Enterprise Networking

- Concurrent Engineering

- Organisational Learning

- Appraisal Methodology

- Manufacturing Information Systems 
Further, we will try to identify whether the work to be done is short term, medium term or long term.

\subsection{Inter Enterprise Networking}

The extended enterprise is an expression of the market driven requirement to embrace external resources in the enterprise without owning them. Core business focus is the route to excellence but product/service delivery requires the amalgam of multiple world class capabilities. Changing markets require fluctuating mixes of resources. The extended enterprise, which can be likened to the ultimate in customisable, reconfigurable manufacturing resource, is the goal. The process is applicable even within large organisations as they increasingly metamorphasise into umbrellas for smaller business units/focused factories.

The operation of the extended enterprise requires take up of communications and database technologies which are near to the current state of the art. However the main challenge is organisational rather than technological. Concerns experienced in the flatter organisations developed in Computer Integrated Manufacturing type business enterprises such as trust, credibility and project management assume a much higher profile in the extended enterprise.

\section{Research and Development Opportunities}

\section{Short Term}

1. A methodology to determine and support the information processes in the extended enterprise. This work will build on process modelling but extend beyond the traditional single business boundary. It will provide the basis for effective extended enterprise operation by defining what information should be communicated and when and where decisions must be made.

\section{Medium Term}

1. Reverse Logistics. Today very sophisticated systems exist to distribute product from the producer to consumers. Virtually no systems exist to return products at end of life. Procedures and supporting systems and tools are needed to enable product return and reuse.

2. Pre Qualification of Partners. The extended enterprise is a volatile environment; partners will regularly join and rejoin a new 'consortium' of enterprises. Pre qualification tools to determine entry level and acceptability are required. These need to embrace physical process and knowledge work capability.

3. Architecture for engineering partnership, an abstract representation of an extended enterprise engineering partnership and associated systems architecture. This would support concurrent engineering across the extended enterprise and, through specification to participating partners, enable realisation of short Time to Market in this environment. 


\section{Long Term}

1. Business and legal framework to facilitate the emergence of transient integrated enterprises. Issues to be researched, understood and articulated include product liability responsibility across the value chain, recyclability issues, ownership of individual enterprises and products, intellectual property rights etc.

\subsection{Concurrent Engineering (CE)}

The UK CE industrial forum has defined Concurrent Engineering as 'delivery of better, cheaper faster products to market by a lean way of working using multidisciplinary teams, right first time methods and parallel processing activities to continuously consider all constraints'. CE must adapt a true life cycle view. This implies consideration of issues such as maintainability, upgradability, recycleability, use of environmentally benign processes, reuse of scarce materials etc. at the earliest stages of design.

Tools in CE can be broadly classified under three categories; management based encompassing such things as team working, project management, and formal methods of geometric manipulation of drawings and models. There has been a focus on tools and techniques and a lack of research into how, when and in what order to implement them. The extended enterprise poses special challenges for the realisation of Concurrent Engineering.

\section{Research and Development Opportunities}

\section{Short Term}

1. New organisational structures for the organisation of concurrent engineering. The distribution of tasks to various members of a product development team is a very important issue. A related question is how to create cross-functional task teams in such a way that:

- $\quad$ traditional accounting procedures do not hamper teamwork;

- the reward system can be based on an individual's performance in projects rather than in the functional organisation;

- $\quad$ reuse of experience is guaranteed;

- learning is part of the task assignment.

In general, the transition from a traditional hierarchical organisation to a networking organisation is especially important for concurrent engineering.

2. Design Tools to Support the Creation of Environmentally Benign Products and Processes. Today we design for manufacture and assembly. In the near future we need to embrace design for disassembly, design for reduced environmental impact, design for reverse logistics, design for reuse and refurbishment, design for a total life cycle etc.

3. Training Systems for use in extended enterprise rapid configuring teams. Multimedia also offers significant potential benefits here. 
Medium Term.

1. Self analysis of design performance. This includes methodologies and systems which prompt the user for information on the design process, map this against established benchmarks and offer specific advice of action, consequences, problems, solutions.

2. Multi function design team support, for use in managing, setting up and participating in extended enterprise design teams.

3. IT tools for the large team environment. Classical CE work has envisaged the compact team. Large multi site, multi sub system design problems require a new infrastructure. Effective operations requires attention to the design process and information management and presentation to minimise information overload. Mutimedia may be effective here.

\section{Long Term}

1. Development of a Negotiation Environment in Concurrent Engineering. This environment will facilitate distributed design activity and will manage the interaction between designers by facilitating appropriate local action.

2. The development of design methodologies to incorporate a true product life cycle view at the earliest (i.e. conceptual) stage in design. [Long Term, Industry and Research Institutes].

\subsection{Organisational Learning}

Competitive advantage is provided by the ability to learn faster than one's rivals. In an environment of uncertainty training does not equip staff with the necessary manufacturing problem solving capacity either in individual or collaborative mode. The central idea of organisational learning is that organisational capability to deal with changes and uncertainty can be fostered, its formal basis defined and best practice replicated and augmented. So in the Learning Organisation, 'organisations', including individuals, can learn from experience to recognise problems and either solve them or initiate successful solution methodologies. In many ways the Learning Organisation adopts the characteristics of a living system. The key difficulties with the concept are what constitutes learning and how to embody it and how to define problems. It is inappropriate to adopt human learning processes and theories directly as a metaphor for the learning which occurs in organisations.

\section{$\underline{\text { Research and Development Opportunities }}$}

\section{Short Term}

1. Formation of a learning process designed to maintain an organisation's competitive advantage, enabling it to selectively customise and develop its own emergent manufacturing techniques. 


\section{Medium Term}

1. Development of a process for managing learning strategically and tactically within an organisation, capitalising on determining the sources of success and failure and hence identifying best practice.

2. Metrics for assessing knowledge generation and use. Knowledge is a key asset of the extended enterprise. Today no widely accepted examples exist with which to measure this important resource.

3. Development of a programme of action-learning research in which the process under development in the previous item is implemented in up to five organisations over a 2year period. This will give the host organisations a chance to be at the leading edge of organisational development through sharing the necessary teaming, problem-solving and learning skills to implement a strategic learning approach within their organisations.

\subsection{Appraisal Methodology within the Extended Enterprise}

Decision support aid in option planning offers help to managers in making effective and defensible decisions about organisation and technology. It concentrates on value rather than mechanism: on why to change manufacturing systems or establish new ones rather than how to do it.

Economic models for manufacturing organisation assume that the two basic forms of combination are markets and hierarchies. In the Extended Enterprise this analysis needs to be revised. Extended enterprises retain the disciplines of the market, yet establish a relationship between supplier and customer that outlasts and goes far beyond single commercial transactions.

Investment Appraisal includes a number of related areas of work. The first, concerns the explanation of various kinds of manufacturing organisation: why supply chains should be integrated within the umbrella of a single firm, how the concentration of suppliers and buyers in a particular market can be explained, where economies and diseconomies of scale lie, and so on. A second area of work covers the development of decision making tools such as scoring and finance models. These are sometimes derived from theories about how people make choices, and sometimes rest simply on the basis of their intuitive appeal. The main difficulty is that the parameters are difficult to estimate with any degree of objectivity. A further area of interest is the analysis of the risk associated with new industrial technologies. Probabilistic models are the more traditional approach. In essence they capture the idea that the future will take one of a number of possible states, and that a decision maker will choose one of a number of alternative courses of action based on their anticipated payoffs in each of those states. Semantic models substitute terms in natural language for both the probabilities of future states occurring and for the payoffs. One example of such work is the AMBITE (Advanced Manufacturing Business Integration Tool for Europe) project with BRITE-EURAM program of the EU. This project is using semantic modelling approaches to analyse the impact of technology investment decisions on the manufacturing performance of an enterprise. 


\section{$\underline{\text { Research and Development Opportunities }}$}

\section{Medium Term}

1. A rigorous methodology to determine the basis of partnership in extended enterprises should be established. This will lead to a prescription of how industrialists need to conduct their business in the resulting organisation.

2. Development of financial value estimating systems. Current financial value estimates are too simplistic and do not properly consider growth options. This is particularly significant in the Extended Enterprise.

\section{$\underline{\text { Long Term }}$}

1. Risk analysis. The organisation structure proposed requires better tools to assess the degree to which qualifications of costs and benefits is subject to error. Simple probabilities are less useful than methodologies which address how people react in the face of uncertainty and how they act to mitigate the effects of uncertainty.

2. Value chain costing and appraisal. The combination of environmental and society concerns relating to manufactured product life cycles and the extended enterprise is a new area. This is likely to have significant impact on the development of competitive and responsive extended enterprise operators.

3. Integrated methodologies. Prescriptive and descriptive models which connect general themes such as economies of scope, environmental impact, with the search for new investment opportunities, with the metrics by which these opportunities are assessed, and the way in which uncertainty about these opportunities can be expressed and managed.

\subsection{Manufacturing Information Systems}

The extended enterprise manufacturing system requires channels that convey information from one system to another. Without these connections the gains in productivity and flexibility that we look for are compromised by mismatches and rigidities at their boundaries.

One form of this problem involves the integration of existing, proprietary applications that have been built on heterogeneous infrastructures: differing machines, operating systems and database management systems. We have to retrofit to relatively impenetrable human and technical systems the mechanisms that will make their operation cohesive in an extended enterprise environment. In particular we have to provide for product traceability and potential liability arising from environmental legislation and value chain costing.

Distributed database mechanisms offer a potential solution. By presenting the data on which applications work in a uniform, self-consistent way we can make sure they share the same view of the factory. Where the same data is stored in more than one of the local systems it should have the same meaning, if not an identical form, in all of them. The object transcrip- 
tion approach, avoids some of the intricacies of distributed databases. The movement of data from one local system to another takes place in complex objects of pre-defined content. Such schemes avoid having to reconcile the different data models and manipulation languages underlying the different applications.

Apart from data infrastructure the extended enterprise, extra enterprise teaming, and prosumer product configuration, require new information communication mechanisms.

Developments in communications technology such as mobile telephones, fax, electronic mail, video conference and even express delivery of conventional mail have been widely adopted. Advances in computer communications have been driven by the need to transfer data from one computer to another. Only electronic mail has attempted to exploit the capability to allow one computer user to communicate directly with another user. Multimedia is the combination of appropriate communications media to best represent human readable information. It can be particularly useful to non specialist users. It allows customer input to the manufacturing system in a novel way. It allows remote site concurrent engineering teams to hold meaningful dialogue. It offers scope for bandwith effective communication of graphical information. It is near language independent.

The potential for multimedia as an extended enterprise user-to-user communications medium is largely unexplored. Most of the physical infrastructure required is being put in place. Telecommunications utilities are proceeding with the implementation of ISDN services, and there is a large base of existing users with demanding communications needs.

\section{Research and Development Opportunities}

\section{Medium Term}

1. Alternative integration methods. Different business situations place differing demands on information channel cost, quality, volume, graphical and textural content. Methodologies appropriate to these environments need to be readily mapped to the need and the implications for business strategy.

2. Integrating remote applications. Methodologies to resolve inconsistent data meanings and structures across the extended enterprise.

3. Authoring tools for manufacturing multimedia messages. Currently comprehensive tools and author expertise are expected, and development time and cost are justified by having many receivers and an extended lifetime for every message. This is not appropriate for a communications application, where there may be one receiver and a short message lifetime.

\section{Long Term}

1. Information Systems to support reverse logistics. In the long term it may be necessary to track each product throughout it's total life cycle and to maintain data on this product to support refurbishment, remanufacturing and ultimately reuse. This presents an enormous challenge to information technology. 
2. Product traceability. The determination of a process to cost effectively allow environmentally sensitive product to be tracked in field. [Medium

3. Product description communication. Methodologies for cost effective transmission and control of appropriate graphical, pictorial and textural data to support the business and product development process. This would allow best use of language independent moving image technology.

\section{CONCLUSIONS}

In this paper we have argued that the Extended Enterprise forms the context for today's research and development efforts in manufacturing systems. We believe that the developments over the past ten years or so in manufacturing systems engineering, including for example Lean Production and World Class Manufacturing facilitate the emergence of the Extended Enterprise. Finally we presented our ideas on the research agenda for the future in manufacturing systems.

\section{REFERENCES}

- Browne, J., Sackett, P.J., Wortmann, J.C. (1993, November), "The System of Manufacturing: A perspective study", Study for DGXII of the EU.

- $\quad$ Browne, J., Sackett, P.J., Wortmann, J.C. (1994, June) "The Extended Enterprise - A Context for Benchmarking", Presented at the IFIP WG5.7 Workshop on Benchmarking - Theory \& Practice in Trondheim, Norway.

- De Meyer, A. (1992, December), "Creating the Virtual Factory" - Report of the 1992 European Manufacturing Futures Survey", INSEAD, France.

- $\quad$ R. Hayes, S. Wheelwright \& W. Clark (1988), "Dynamic Manufacturing - Creating the Learning Organisation", Collier MacMillian, London.

- M. Hammer \& J. Champy (1993), "Re-engineering the Corporation - A Manifesto for Business Revolution", Nicholas Brealey Publishing.

- D.T. Jones (1992): "Beyond the Toyota Production System : the era of Lean Production" in "Manufacturing Strategy - Process and Content", Chapman \& Hall, 1993, Editor C. Voss.

- R.J. Schonberger (1990), "Building a Chain of Suppliers", Hutchinson Business Books, USA. 
- Tipnis, V.J. (1993), "Evolving Issues in Product Life Cycle Design", Annals of the CIRP, Volume 42, No.1.

\section{BIOGRAPHY}

Professor Jim Browne is Director of the CIM Research Unit (CIMRU) at University College Galway in Ireland. He has been engaged as a consultant by companies such as Digital Equipment Corporation, Westinghouse Electric, Apple Computers, De Beers, AT Cross and a number of Irish manufacturing companies, to work in areas such as manufacturing strategy, manufacturing systems simulation and production planning and control. He has also been engaged by the European Union to work on the development of the ESPRIT programme (DGXII) and the BRITE programme (DGXII). He has been engaged in EU research projects with industrial companies such as COMAU in Italy, Renault in France, DEC in Munich, Alcatel in France and a number of European Universities, including the University of Bordeaux, the University of Eindhoven, the University of Berlin and the University of Trondheim.

For the past two years, he has been engaged as a consultant on a project funded jointly by the EU and the State Scientific and Technical Committee of China (SSTCC) - (Peoples Republic of China) to support the National CIMS (Computer Integrated Manufacturing Systems) project of China.

\section{Note :}

The authors would like to thank Mr Antonio Colaco of DGXII in the Commission of the European Union for insightful comments on an earlier draft of this paper. 